\title{
Parameter Analysis on Torque Stabilization for the Eddy Current Brake: A Developed Model, Simulation, and Sensitive Analysis
}

\author{
Quan Zhou, ${ }^{1,2,3}$ Xuexun Guo, ${ }^{1,2,3}$ Gangfeng Tan, ${ }^{1,2,3}$ Xiaomeng Shen, \\ Yifan Ye, ${ }^{3}$ and Zhaohua Wang ${ }^{3}$ \\ ${ }^{1}$ Hubei Key Laboratory of Advanced Technology for Automotive Components, Wuhan University of Technology, Wuhan, \\ Hubei 430070, China \\ ${ }^{2}$ Hubei Collaborative Innovation Center for Automotive Components Technology, Wuhan, Hubei 430070, China \\ ${ }^{3}$ School of Automotive Engineering, Wuhan University of Technology, Wuhan, Hubei 430070, China \\ ${ }^{4}$ School of Economics, Wuhan University of Technology, Wuhan, Hubei 430070, China \\ Correspondence should be addressed to Gangfeng Tan; 18995570176@189.cn
}

Received 10 March 2015; Revised 15 May 2015; Accepted 17 May 2015

Academic Editor: Emiliano Mucchi

Copyright (C) 2015 Quan Zhou et al. This is an open access article distributed under the Creative Commons Attribution License, which permits unrestricted use, distribution, and reproduction in any medium, provided the original work is properly cited.

\begin{abstract}
Eddy current brake (ECB) is an attractive contactless brake whereas it suffers from braking torque attenuation when the rotating speed increases. To stabilize the ECB's torque generation property, this paper introduces the concept of anti-magneto-motive force to develop the ECB model on the fundamental of magnetic circles. In the developed model, the eddy current demagnetization and the influence of temperature which make the braking torque attenuation are clearly presented. Using the developed model of ECB, the external and internal characteristics of the ECB are simulated through programming by MATLAB. To find the sensibility of the influences on ECB's torque generation stability, the stability indexes are defined and followed by a sensibility analysis on the internal parameters of an ECB. Finally, this paper indicates that (i) the stability of ECB's torque generating property could be enhanced by obtaining the optimal combination of "demagnetization speed point and the nominal maximum braking torque." (ii) The most remarkable influencing factor on the shifting the demagnetization speed point of ECB was the thickness of the air-gap. (iii) The radius of pole shoe's cross section area and the distance from the pole shoe center to the rotation center are both the most significant influences on the nominal maximum braking torque.
\end{abstract}

\section{Introduction}

Eddy current brake (ECB) is an attractive auxiliary braking device for vehicles, which could be directly controlled by wire. Comparing with some other auxiliary brake, ECB enjoys the following advantages [1-3]: (a) the ECB is easy to be controlled, and it nearly does not suffer the braking delay, for it is directly controlled by the current applied in the wire. (b) The ECB enjoys better performances in low-speed domain than either regenerative brake or hydraulic retarder. (c) The ECB does not require the internal combustion engine (ICE) to provide the negative pressure as the pneumatic source (nevertheless, the hydraulic retarder and the engine brake need the internal combustion engine to provide negative pressure when they are working). Overall, the ECB should be a promising auxiliary brake for future vehicles, as vehicles tend to be more electric drove, especially for the vehicles without internal combustion engine like pure electric vehicles and fuel cell battery electric vehicles.

For the ECB, the applied current generates magnetic flux in the core, and an eddy current is induced around pole shoe of the core when the magnetic flux goes through a rotating conductive disk. The braking force is generated by the interaction between eddy current and magnetic flux. According to the theoretic works in $[4,5]$, the braking torque of an ECB was simply expressed as a function of the angular speed of a disk and the applied current when ignoring the demagnetization. If the angular speed is a constant, the 


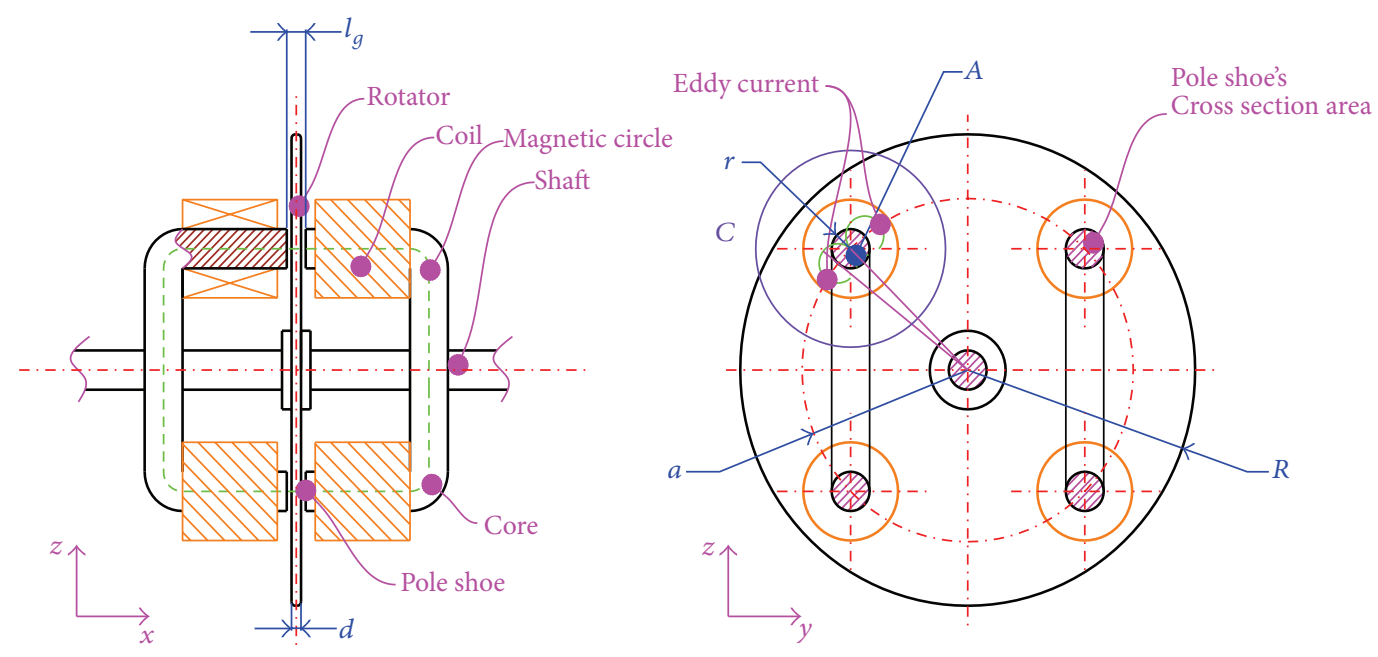

FIGURE 1: Schematic of a typical ECB.

braking force is proportional to the applied current, and vice versa. Actually, the braking torque would not be continuously increasing while the angular speed or applied current rises. According to experimental results on ECB's torque-speed property [2, 6-8], at the very beginning, the braking torque increases rapidly while the rotating speed ascends. Nevertheless, after the braking torque peaked at its maximum value, the braking torque tends to drop significantly if the rotating speed goes on increasing. The reason of this phenomenon is that the demagnetization effect cut down the ECB's torque generation property while, at the same time, the ECB's torque stabilization is destroyed. Specifically, for excitation eddy current brake, to prevent the demagnetization effect not only means to improve the braking stability but also to save the energy. To explore the electromagnetic property of the ECB, Smythe [1] firstly implies magnetic potential theory to model the ECB, and he described the demagnetization effects by deriving the Maxwell's equations. Wouterse [3] indicates structure index $\mathrm{C}$ based on the numerical analysis and experiment validation to simplified Smythe's model. Simeu and George's firstly [4] introduced an ECB based on magnetic circle theory and his model is widely used in control algorithm research on ECB $[5,9]$. However, Simeu's model can only express how ECB retards the rotator in low-speed region, but it cannot fully describe the ECB's property in highspeed region for it ignored the eddy current demagnetization. Recently, with the development of computational science, many have tried to model ECB with FEM [10-12], although the FEA results could be a reference for engineering design, it is still reasonable to develop current ECB's theoretical model for detecting the influences on ECB's braking torque stability [6-8]. Hence, this paper presents a modelling method based on magnetic circle theory, which is easy to find the relations of the internal parameters to the braking torque stabilization. Using this developed ECB model, the sensitivity of the influences of the braking torque stabilization could be easily found through simulation and related sensitivity analysis.

This paper is organized as follows. Section 2 presents the basic construction of a typical ECB while the parameters for modeling are present. Section 3 introduces the modeling assumptions and the mathematical modeling works; in this section, by introducing the concept of antimagnetic force, a developed ECB model is derivate in detail. Section 4 includes the simulation results on both external parameters and internal parameters of the ECB based on the developed model. In Section 5, the braking torque stability index including the demagnetization speed point (demagnetization speed point) and the nominal maximum braking torque is introduced, followed by a sensibility analysis on internal parameters to braking torque stability index.

\section{The Principle of ECB}

The configuration of a typical ECB is presented in Figure 1. A typical ECB consisted of rotator, shaft, coil, and iron core. For easy fabrication, this paper presents an ECB that is designed with two pairs of coil-core systems which are circumferentially equispaced around the rotator. The rotator is a copper made disc, with the radius of $R$ and the thickness of $d$. In each coil-core system, two U-shaped iron cores are symmetry arranged aside the rotator. The zone between these two U-shaped iron cores calls air-gap; the thickness of the airgap is $l_{g}$. At the heat of each iron core, the coils are winding into N/8 turns and each coil is series-wound connected. The pole shoe is at the top of the U-shaped iron-core, and in this paper, the pole shoe's cross section area is roundness with radius of $r$ and the area of $A$.

Figure 2 displays how the eddy currents are generated and how the ECB retards the vehicle. The applied current generates magnetic flux in the core (see the green dotted circle in Figure 1), and an eddy current (see the green solid circle in Figures 1 and 2) is induced around pole shoe (the pole area of the core, see Figure 1) of the core when the magnetic flux goes through a rotating conductive disk. The braking force (its direction is negative to the rotating speed, see Figure 2) is generated by the interaction between eddy current and magnetic flux. The braking torque generated by ECB is then 


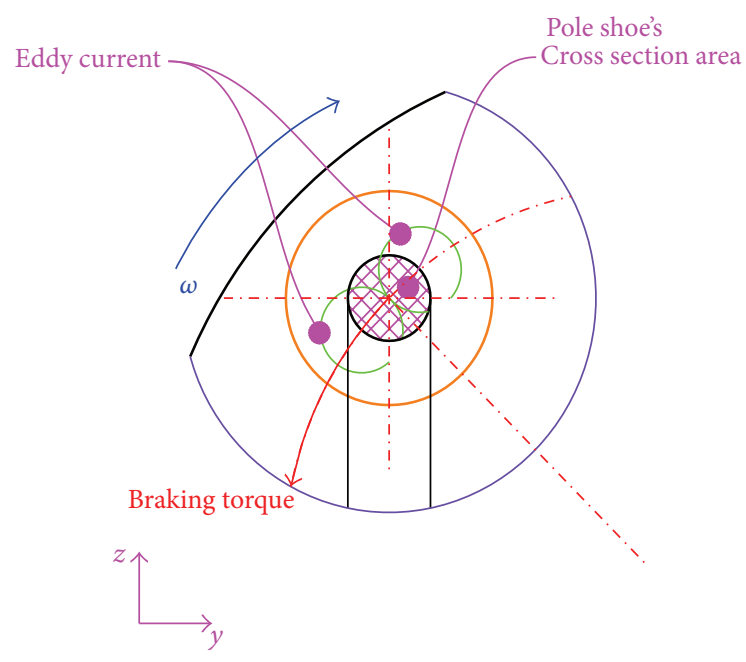

FIGURE 2: Working principle of a typical ECB.

transferred to the wheels through the driving shaft and final reducing gear.

\section{Mathematical Modeling of the ECB}

3.1. Simplifying Equivalent Magnetic Circle and Assumptions. According to Ampere-Maxwell equation, the magnetic field intensity generated by the applied current could be calculated as

$$
\oint_{l} H d l=I_{\text {net }} .
$$

In one pair of the coil-corn systems, the path of integration is a closed circle, and hence, in one pair of the coil-corn system, $\oint_{l} H d l=H \cdot l$, where $l$ is the equivalent length of the magnetic circle and $H$ is the magnetic field intensity. $I_{\text {net }}$ is the overall current around the magnetic circle and it is calculated as $I_{\text {net }}=N \cdot i$, where, $N$ is the turns of the coil and $i$ is the applied current in the coil.

When given the definition of magnetic flux density $B=$ $\mu H$, the overall magnetic flux in one pair of the coil-corn system $\Phi$ is calculated as

$$
\Phi=B A=\frac{\mu N i A}{l} .
$$

When defining the magnetic force as $\mathscr{F}=I_{\text {net }}$, $A$ as the crosssection area of the pole shoe, and the reluctance as $\mathscr{R}=l / \mu A$, the basic magnetic theory is established:

$$
\Phi=\frac{\mathscr{F}}{\mathscr{R}} .
$$

In (3), $\mathscr{F}$ is combined magnetic force, which is the sum of magnetic force and antimagnetic force. $\mathscr{R}$ is the overall reluctance, which could be calculated as the overall resistance in electric circles.

According to magnetic circle theory, the equivalent magnetic circle of ECB is established in Figure 3.
The modeling work on ECB is based on its equivalent magnetic circle; for further derivation, this paper presents the following assumptions.

(1) The air-gap is acceptable thick that the cross-section area of magnetic flux in the rotator could be estimated as the cross section area of the pole shoe; namely, $A=$ $\pi r^{2}$.

(2) In the rotator, the reluctance in shaft direction is $\mathscr{R}_{d_{a x}}^{*}=d / \mu_{0} \pi r^{2}$, while the reluctance in radius direction is $\mathscr{R}_{d_{r a}}^{*}=a / \mu_{0} r d$. Here assume that $a \gg d$, so that $\mathscr{R}_{d_{r a}}^{*} \gg \mathscr{R}_{d_{a x}}^{*}$ and all the magnetic flux could cross through rotator disc from the shaft direction.

(3) The influence of the radius of the rotator disc $R$ is ignored as this factor only affects the value of the braking torque but not the stability of the braking torque, and the influence of this factor on the braking torque is discussed in some presented works before; see $[1,3,13]$.

(4) The braking time is acceptable short that the temperature variation could be ignored.

Hence, the simplified equivalent magnetic circle of ECB (see Figure 4 ) could be rebuild as a series-wound magnetic circle with magnetic force $\mathscr{F}_{i}$, air-gap reluctance $\mathscr{R}_{a}$, antimagnetic force generated by eddy current in the rotator $\mathscr{F}_{\text {eddy }}$, and iron core reluctance $\mathscr{R}_{c}$.

3.2. Derivation of the ECB Model. According to the simplified equivalent magnetic circle, the magnetic flux in one coil-core system is calculated as

$$
\Phi_{c}=\frac{\mathscr{F}_{i}-\mathscr{F}_{\text {eddy }}}{\mathscr{R}_{c}+\mathscr{R}_{a}+\mathscr{R}_{d}},
$$

where $\mathscr{F}_{i}$ is the magnetic force generated by applied currents in the coil and $\mathscr{F}_{\text {eddy }}$ is the defined antimagnetic force provided by eddy current in the rotator. $\mathscr{R}_{c}, \mathscr{R}_{a}$, and $\mathscr{R}_{d}$ are the reluctance value of coil, air-gap, and rotator disc, respectively.

The reluctance of the air-gap could be calculated as

$$
\mathscr{R}_{a}=\frac{l_{g}-d}{\mu_{0} A},
$$

where $\mu_{0}$ is the permeability of vacuum with the value $4 \pi \times$ $10^{-7} . l_{g}$ is the distance between two pole shoes and $d$ is the thickness of rotator disc and $A$ is the cross section area of the pole shoe.

In this paper, the rotator is made up of copper; hence its permeability is similar to the permeability of vacuum, and hence, we rewrote the reluctance outside the iron corn as

$$
\mathscr{R}_{g}=\mathscr{R}_{a}+\mathscr{R}_{d}=\frac{l_{g}}{\mu_{0} A} .
$$

The reluctance of the core could be calculated as

$$
\mathscr{R}_{c}=\frac{l_{c}}{\mu_{c} \mu_{0} A} .
$$




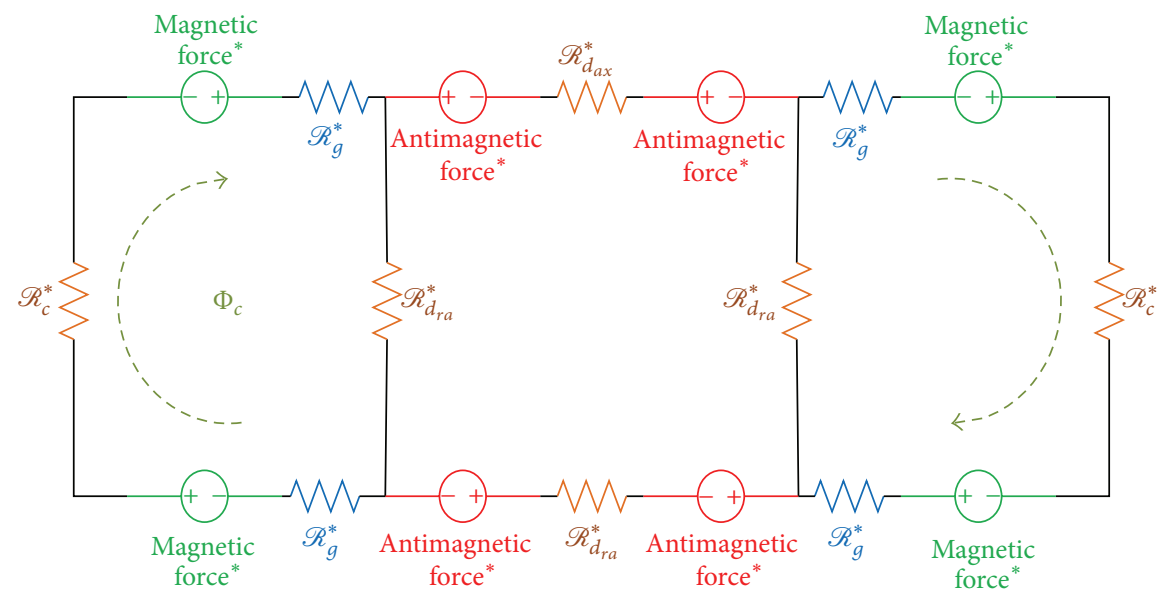

FIgURE 3: Diagram of the equivalent magnetic circle of ECB.

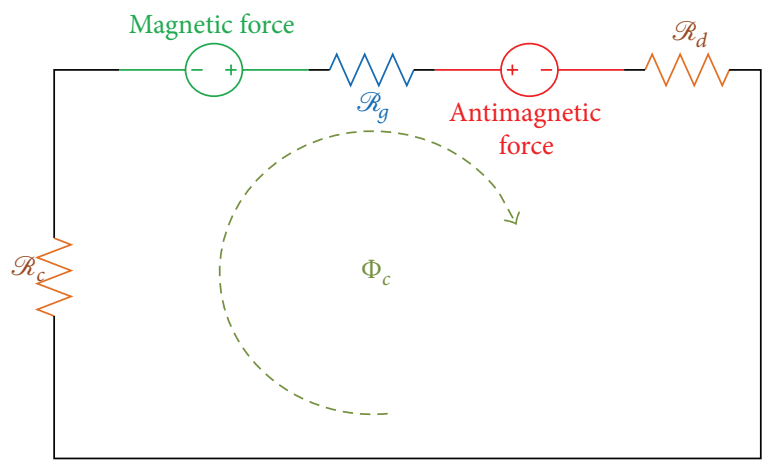

Figure 4: Diagram of the simplified equivalent magnetic circle of ECB.

In (7), $l_{c}$ is the length of the equivalent magnetic circle in iron core. $\mu_{c}$ is relative permeability of ferromagnetic material. The value of $\mu_{c}$ is around 6000 so that the value of $\mathscr{R}_{c}$ is so much less than $\mathscr{R}_{g}$, and hence, it could be neglected in (2).

Then, (4) could be rewritten as

$$
\Phi_{c}=\frac{\mathscr{F}_{i}-\mathscr{F}_{\text {eddy }}}{\mathscr{R}_{g}},
$$

where the magnetic force $\mathscr{F}_{i}$ could be calculated by multiplying the turns of coil $N$ and the applied current in the coil $i$ :

$$
\mathscr{F}_{i}=N i .
$$

In addition, the defined antimagnetic force $\mathscr{F}_{\text {eddy }}$ generated by eddy current in the rotator disc could be calculated as

$$
\mathscr{F}_{\text {eddy }}=\oint_{s} J_{e} d s=J_{e} \cdot d \cdot r .
$$

Here $J_{e}$ is the current density of eddy current in the rotator disc:

$$
J_{e}=\sigma \cdot a \cdot\left(\omega \times B_{c}\right)
$$

In (8), $a$ is the distance from the center of pole shoe area to the rotating center and $B_{c}=\Phi_{c} / A=\Phi_{c} / \pi r^{2}$ is the magnetic flux density in one pair of the coil-core system.

Hence, (10) could be rewritten as

$$
\begin{aligned}
\mathscr{F}_{\text {eddy }} & =\sigma \cdot a \cdot\left(\omega \times \frac{\Phi_{c}}{\pi r^{2}}\right) \cdot d \cdot r \\
& =\sigma \cdot a \cdot d \cdot\left(\omega \times \frac{\Phi_{c}}{\pi r}\right) .
\end{aligned}
$$

According to (4), the antimagnetic force could be also calculated by

$$
\mathscr{F}_{\text {eddy }}=N i-\Phi_{c} \cdot \mathscr{R}_{g} .
$$

From (12) and (13), the magnetic flux in one coil-core system is obtained as

$$
\Phi_{c}=\frac{N i}{\left(\mathscr{R}_{g}+(\sigma a d \omega / \pi r)\right)}
$$

Hence, the magnetic flux density in this coil-core system is

$$
B_{c}=\frac{\Phi_{c}}{\pi r^{2}}=\frac{N i}{\left(\left(l_{g} / \mu_{0}\right)+\sigma a d r \omega\right)} .
$$

Then (11) could be rewritten as

$$
J_{e}=\sigma \cdot a \cdot \omega \times\left(\frac{N i}{\left(l_{g} / \mu_{0}\right)+\sigma a d r \omega}\right) .
$$

The total power dissipation may be calculated simply by integrating $\rho \cdot J_{e}{ }^{2}$ over the cylindrical volume $\pi r^{2} \cdot d[3,14,15]$, where $d$ and $r$ denote, respectively, the disc thickness and the radius of a circle with the same area as the pole face. Therefore, the total dissipation is

$$
P_{\mathrm{diss}}=\rho \cdot J_{e}^{2} \cdot \pi r^{2} \cdot d
$$


From view of energy follow Theory, the dissipated energy in eddy current is equal to the dissipated kinetic energy of vehicle, and hence, the braking torque could be evaluated as

$$
T_{b}=\frac{P_{\text {diss }}}{\omega}=\sigma a^{2} \pi r^{2} d\left(\frac{N}{\left(l_{g} / \mu_{0}\right)+\sigma a d r \omega}\right)^{2} i^{2} \omega
$$

In addition, $\sigma$ is the conductivity of the rotor material and it could be calculated as

$$
\sigma=\frac{1}{\rho_{0}(1+\alpha t)} .
$$

Here, $t$ is the temperature of the rotating disc. $\rho_{0}$ and $\alpha$ are electrical resistivity at $0^{\circ} \mathrm{C}$ and temperature coefficient of resistance, respectively. For a copper made rotator disc, $\rho_{0}=1.75 \times 10^{-8} \Omega \mathrm{m}$ and $\alpha=4.1 \times 10^{-3} 1 /{ }^{\circ} \mathrm{C}$. Hence, (18) could be developed into

$$
\begin{aligned}
& T_{b}=\pi r^{2} a^{2} d\left(\frac{N \mu_{0}}{l_{g} \rho_{0}^{2}(1+\alpha t)^{2}+a d r \omega \rho_{0}(1+\alpha t)}\right)^{2} \\
& \quad \cdot i^{2} \omega .
\end{aligned}
$$

\section{Simulation on ECB}

4.1. Simulation on the External Characteristics of the ECB. Simulations are performed on the developed mathematical model of braking torque in ECB, by means of MATLAB.

For a typical commercial vehicle, which is equipped with an ECB in its driving shaft, the ECB rotator's rotating speed is highly related to the vehicles driving speed. Their relationship is $n_{\mathrm{ECB}}=\left(v_{\text {vehilce }} / r_{\text {wheel }}\right) i_{0}$, where $v_{\text {vehicle }}$ is the vehicle's driving speed, $r_{\text {wheel }}$ is the radius of the rear-wheel, and $i_{0}$ is the driving ratio of the final reducing gear. According to China's transportation laws, in most highways, the maximum speed of commercial vehicle is limited to $80 \mathrm{~km} / \mathrm{h}$, and hence, in the simulation, the rotator's rotating speed is set from 0 to $2500 \mathrm{rpm}$. Simulations on the braking torque of ECB were carried out at the speed from 0 to $2500 \mathrm{rpm}$, the applied current was set from 0 to $20 \mathrm{~A}$, and the temperature of rotator disc was set from 0 to $800^{\circ} \mathrm{C}$ with the parameters employed in simulations listed in Table 1.

Figure 5 shows the ECB's torque versus rotating speed property when the current is kept constant in $10 \mathrm{~A}$ and the temperature of the rotator disc is $200^{\circ} \mathrm{C}$; the simulation is based on the assumption that the influence of temperature transient variation on the braking torque is ignored. The simulation result indicates that the working condition of an ECB could be divided into two domains, namely, lowspeed domain and high-speed domain. The properties in both domains are acceptable corresponding to the bench test results present in $[1,13]$. In low-speed domain, the braking torque is proportional to the rotator's rotating speed. Nevertheless, the proportional relationship is broken as the speed increases. There is a peak value on the curve, and the abscissa of this peak value is a demagnetization speed point. The ECB came to its high-speed domain when the rotating
TABLE 1: Model parameters of ECB.

\begin{tabular}{lcc}
\hline Parameter & Value & Unit \\
\hline$a$ & 85 & $\mathrm{~mm}$ \\
$d$ & 3 & $\mathrm{~mm}$ \\
$r$ & 20 & $\mathrm{~mm}$ \\
$N$ & 720 & turns \\
$l_{g}$ & 16 & $\mathrm{~mm}$ \\
$\mu_{0}$ & $4 \pi \times 10^{7}$ & $\mathrm{H} / \mathrm{m}$ \\
$\rho_{0}$ & $1.75 \times 10^{-8}$ & $\Omega \mathrm{m}$ \\
$\alpha$ & $4.1 \times 10^{-3}$ & $1 /{ }^{\circ} \mathrm{C}$ \\
\hline
\end{tabular}

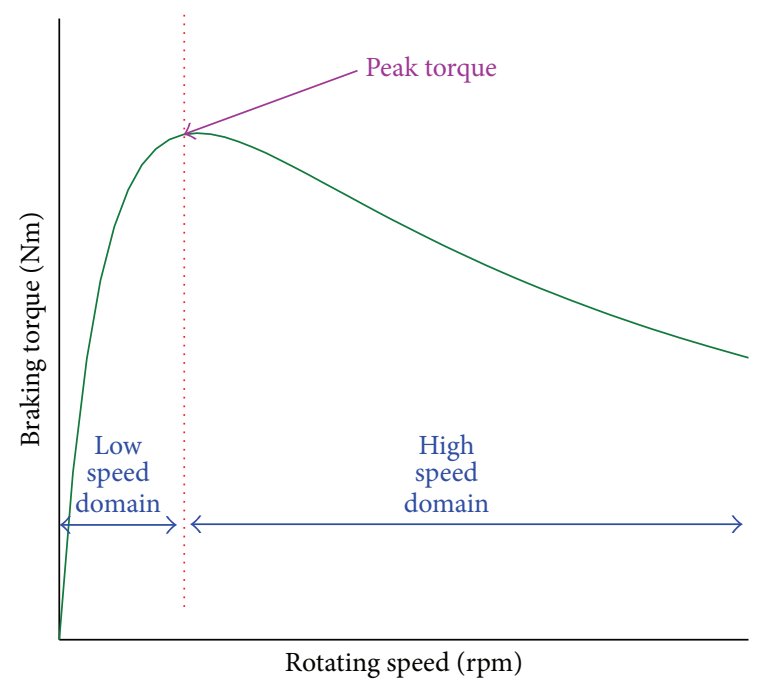

Figure 5: Simulation result of torque-speed property of ECB.

speed surpasses the demagnetization speed point, and in high-speed domain, the braking torque and the rotating speed have an inverse proportional relation.

From the simulation result presented in Figure 6, both the applied current and the rotating speed affected the braking performance of ECB. In low-speed domain, the braking torque tended to be increasing while applied current or rotating speed was arising. Nevertheless, when the rotating speed surpasses a nomination value, the braking torque attenuates dramatically, and the reducing rate of the torque attenuates is proportional to the applied current, which means if the braking torque starts to attenuate, the braking torque could not be stalely controlled by simply changing the applied current.

The temperature also affects the torque-speed characteristics of ECB. Figure 7 shows the braking torque characteristics versus both temperature and applied current; the braking torque tended a significantly attenuation while the temperature of rotator disc is continuously increasing. Deserve to be mentioned, the developed model in this paper maybe circumscribed to fully detecting the influence of temperature on the braking torque, especially the barking torque characteristics versus both temperature and rotating speed. The further developing model should consider the thermal dynamics and this paper just simply observes 

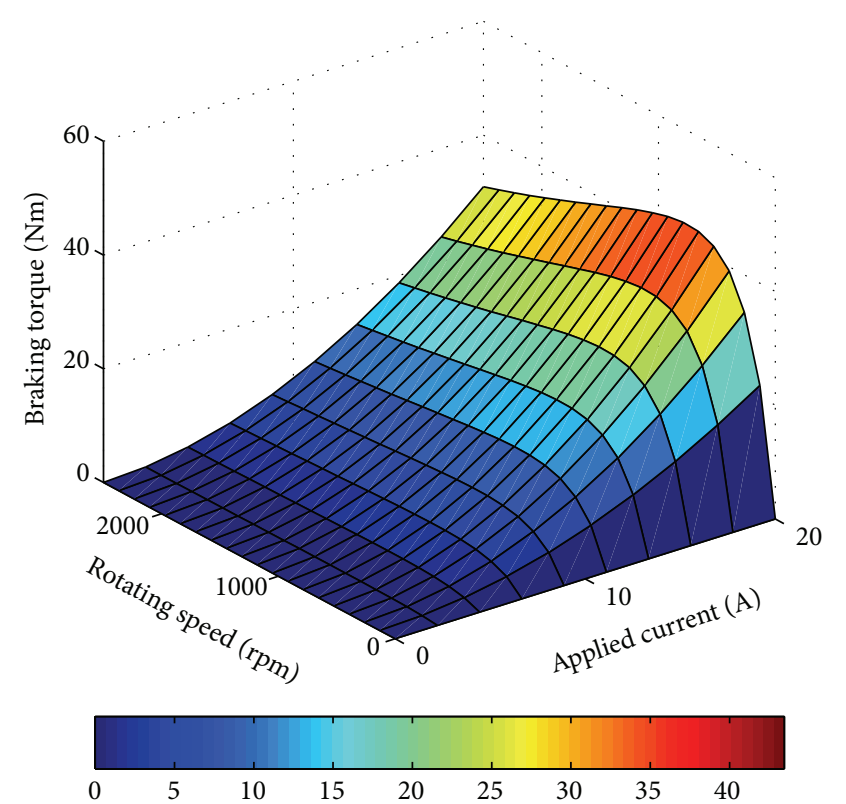

FIGURE 6: Simulation result of braking torque characteristics (rotating speed and applied current).

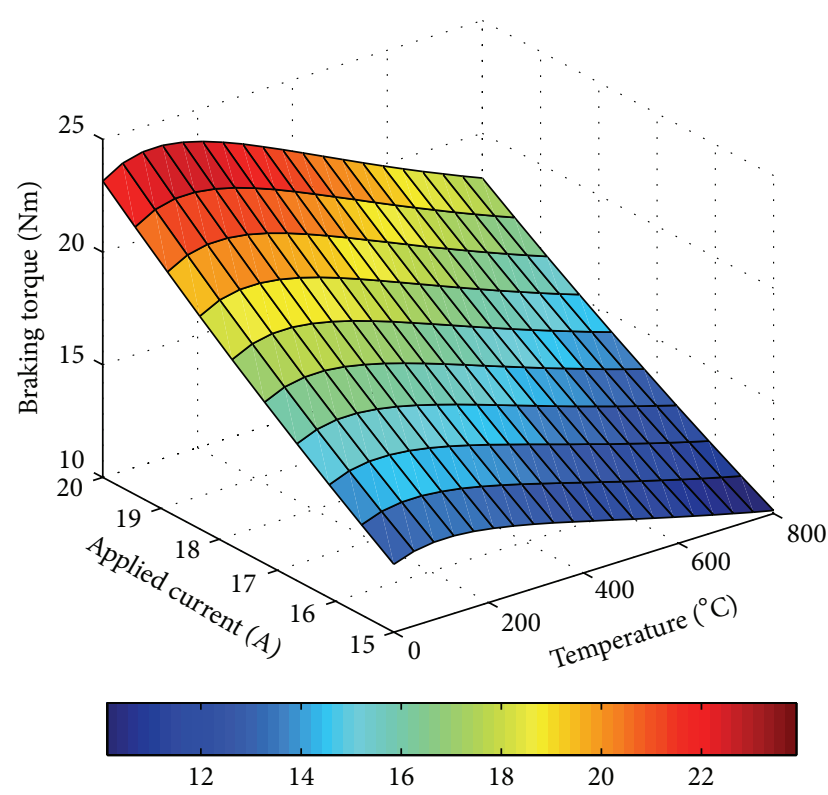

Figure 7: Simulation result of braking torque characteristics (applied current and temperature).

the braking torque characteristic versus temperature in the view of qualitative analysis.

4.2. Simulation on the Internal Characteristics of the ECB. According to the derived mathematical models of braking torque in ECB, the influencing parameters on the ECB's braking torque stability are: the radius of the pole shoe's cross-section area, distance from the pole shoe's area center to the rotating center, thickness of the rotator disc, the thickness of the air gap, and so forth. With variations of

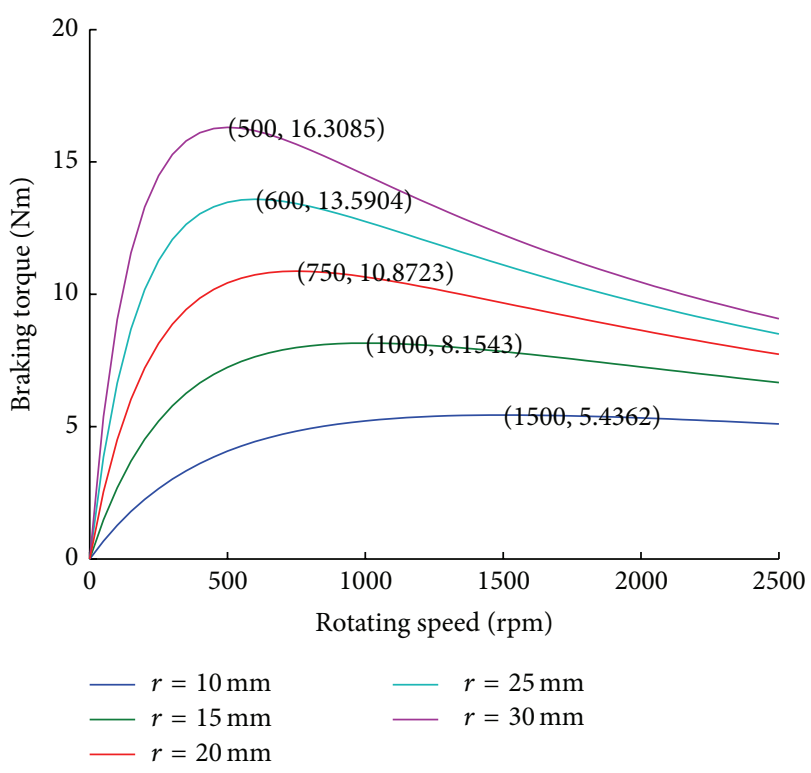

FIGURE 8: Influence of the equivalent radius of pole shoe area.

parameters described above, the influences on ECB torquespeed property were studied, as the applied current is $10 \mathrm{~A}$ and the rotator disc temperature is $200^{\circ} \mathrm{C}$.

As described in Figure 8, the increasing ratio of braking torque in low-speed domain increased with the rise of the radius of the pole shoe's cross section area, while the attenuation ratio in high-speed domain is also increased with the rise of the radius of the pole shoe's cross section area. When the value of the radius of the pole shoe's cross section area increases, the value of the peak torque is increasing, whereas the demagnetization speed point is declining.

As shown in Figure 9, the ECB's torque-speed enjoys a similar tendency while changing the distant from the pole shoe's center the rotating center.

In Figure 10, the increasing ratio of braking torque in lowspeed domain drops with the rise of the thickness of the air gap, while the attenuation ratio in high-speed domain is also damping with the rise of the thickness of the air gap. When the value of the thickness of the air gap increases, the value of the peak torque is descending, whereas the demagnetization speed point is aggrandizing.

Figure 11 indicates that in the low-speed domain the increasing ratio of braking torque arises with the rise of the thickness of the rotator disc, while the attenuation ratio in high-speed domain is also increasing with the rise of the thickness of the rotator disc. While the value of the thickness of the rotator disc is increasing, the value of the peak torque does not change significantly, whereas the demagnetization speed point is aggrandizing.

Conclusively, the simulations indicate that the internal parameters can affect the ECB's torque generating property. For a typical ECB, before the rotating speed surpasses a nominal value, the braking torque provided by the ECB is proportional to the rotating speed. In this domain, the torque could be controlled stably by changing value of applied 


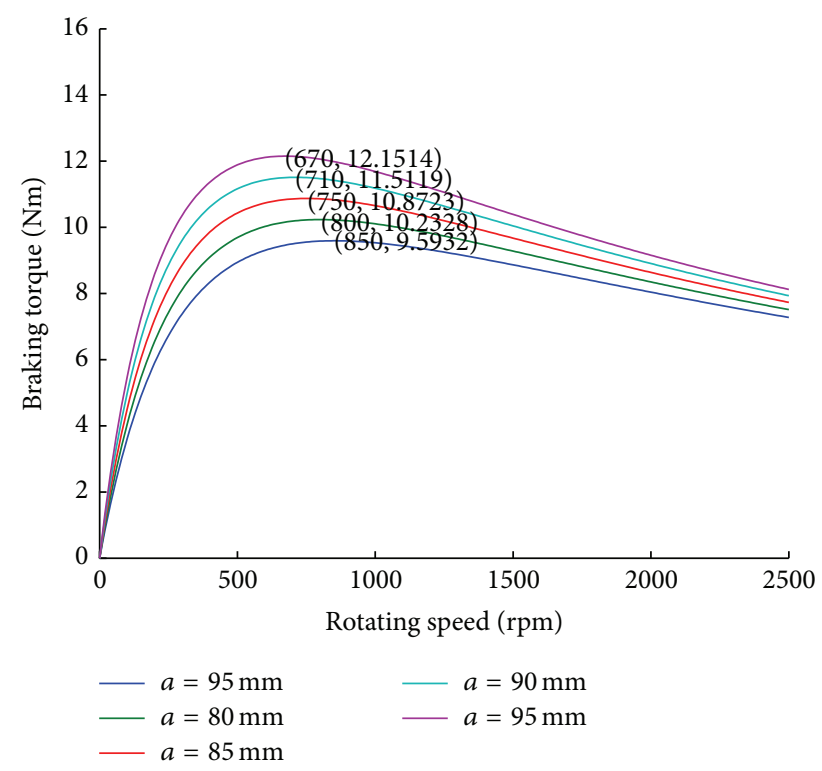

FIGURE 9: Influence of the location of the pole shoe.

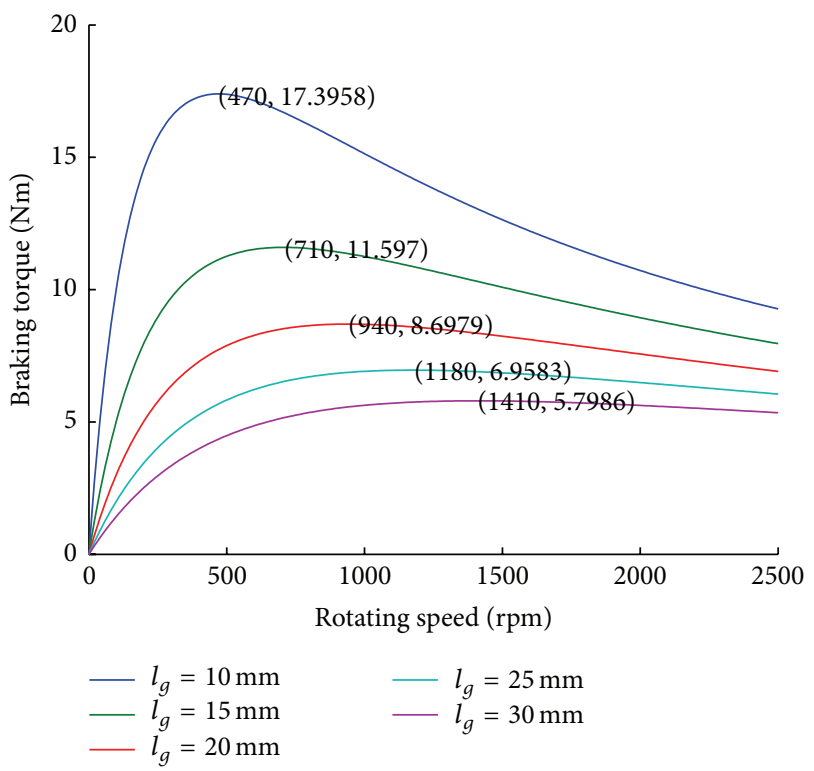

FIGURE 10: Influence of different air-gap thickness.

current; namely, when the applied current is increasing, both the value and the increasing ratio of ECB's braking torque can increase. On the contrary, when the rotating speed goes on increase, the braking torque cannot be simply controlled by changing the applied current as a proportional principle; namely, when the applied current is increasing, the braking torque increases whereas the increasing ratio drops while the rotation speed arises. Hence, to enhance the stability of the braking torque performance, it is should find an optimal combination of ECB's demagnetization speed point and the nominal maximum braking torque, which is defined as the torque stabilization indexes, by optimizing the ECB'S internal parameters.

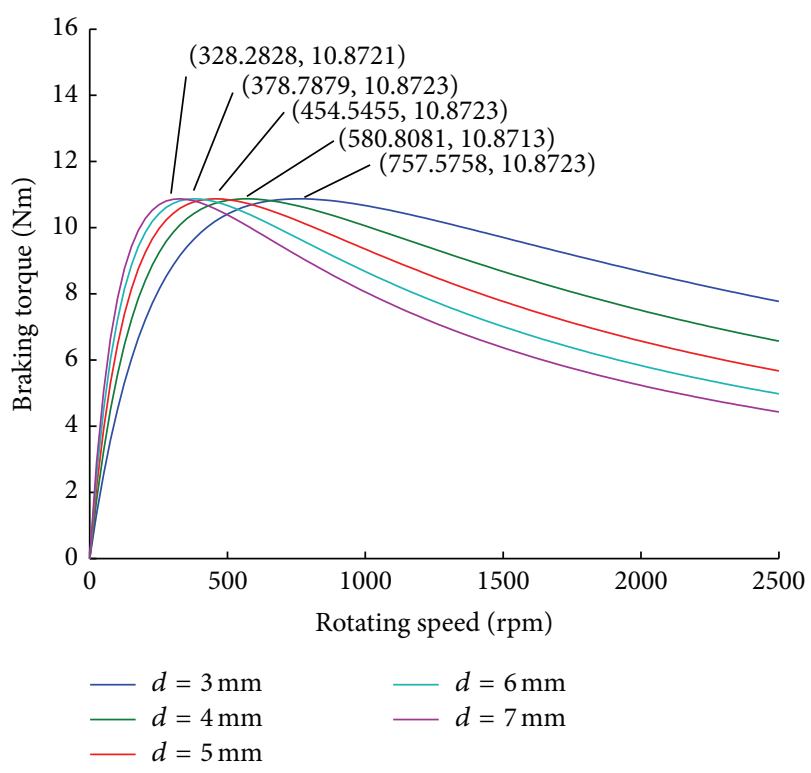

FIGURE 11: Influence of different rotator disc thickness.

\section{Sensibility Analysis on Influences of Torque Stabilization}

To investigate the torque stabilization indexes, this paper firstly introduces the concept of demagnetization speed point, and it is judged by the demagnetization speed point, which could be found as the root of the partial differential equation followed:

$$
\frac{\partial T_{V}}{\partial \omega}=0
$$

Hence, the demagnetization speed point is described as a function of distance from pole shoe center to the rotation center $a$, thickness of the rotator disc $d$, thickness of the air-gap $l_{g}$, and the equivalent radius of the pole shoe's cross section area $r$ :

$$
\omega_{\mathrm{de}}=\frac{l_{g}}{\mu_{0} \sigma a d r} .
$$

In the demagnetization speed point, the nominal maximum braking torque of the ECB could be obtained, and it could be calculated as

$$
\begin{aligned}
T_{\max }= & \pi r^{2} a^{2} d \rho_{0}(1+\alpha t)\left(\frac{N \mu_{0}}{l_{g}+a d r\left(l_{g} / \mu_{0} a d r\right)}\right)^{2} i^{2} \\
& \cdot \frac{l_{g}}{\mu_{0} a d r} .
\end{aligned}
$$

Owing to the structure design, the thickness of the rotator disc is highly $d$ related to the thickness of the air-gap $l_{g}$, and they must follow $d<l_{g}$; hence, in the sensitive analysis, this paper observed the influence of the parameter $l=l_{g}-d$.

According to the simulations in Section 4, both the demagnetization speed point and the nominal torque affect 
TABLE 2: Initial value setting for sensitive analysis.

\begin{tabular}{lcc}
\hline Parameters & Initial value & Unit \\
\hline$a_{0}$ & 90 & $\mathrm{~mm}$ \\
$d_{0}$ & 3 & $\mathrm{~mm}$ \\
$l_{0}$ & 13 & $\mathrm{~mm}$ \\
$r_{0}$ & 20 & $\mathrm{~mm}$ \\
\hline
\end{tabular}

the ECB's braking performance; hence, this paper introduces a two-dimensional braking torque stability index that is made up with demagnetization speed point and nominal torque. With the purpose to investigate the effects of the parameters of ECB on braking torque stabilization, the related sensitivity analysis is carried out. The sensitivities of braking torque stabilization indexes to the parametric variations of ECB were detected using one parametric variation method with the observed parameters including charging the radius of the pole shoe's cross section area, distance from the pole shoe's area center to the rotating center, thickness of the rotator disc, the thickness of the air gap, and so forth. At each measurement, the selected parameter was increased by $5 \%$ of the initial value, while other parameters kept invariable. Then, the variations of demagnetization speed point and the nominal maximum braking torque with the selected parameter were investigated. The sensitivity can be defined as [16]

$$
S=\left|\frac{\Delta y / y_{0}}{\Delta x / x_{0}}\right|,
$$

where $S$ is the sensitivity of index to the selected parameters, $\Delta y$ is the variation of index, $\Delta x$ is the variation of the selected parameter, $x_{0}$ is the initial value of the parameters, and $y_{0}$ is the initial value corresponding to the situation when $x=x_{0}$. The larger the sensitivity value, the more significant the effects of parameter on the evaluation of demagnetization speed point and maximum braking torque.

Using the initial value settings in Table 2, Figures 12 and 13 display the sensitivities of ECB performance to various parameters, and the calculated values of the sensitivity are listed in Tables 3 and 4, respectively.

From Figures 12 to 13 and Tables 3 to 4 , the following conclusions could be obtained. (i) In terms of the effects on shift the demagnetization speed point, the influencing parameters with the sensibility ranked in a descending order were the thickness of the air-gap, the radius of pole shoe's cross section area, the distance from the pole shoe center to the rotation center, and thickness of the rotator disc. (ii) In terms of the nominal maximum braking torque, the radius of pole shoe's cross section area and the distance from the pole shoe center to the rotating center both were of most significant; the thickness of the air-gap was ranked the second place while the influence of the thickness of the rotator disc could be ignored.

\section{Conclusions}

In the presented work, a design scheme of ECB was introduced, and the mathematical model of ECB was developed
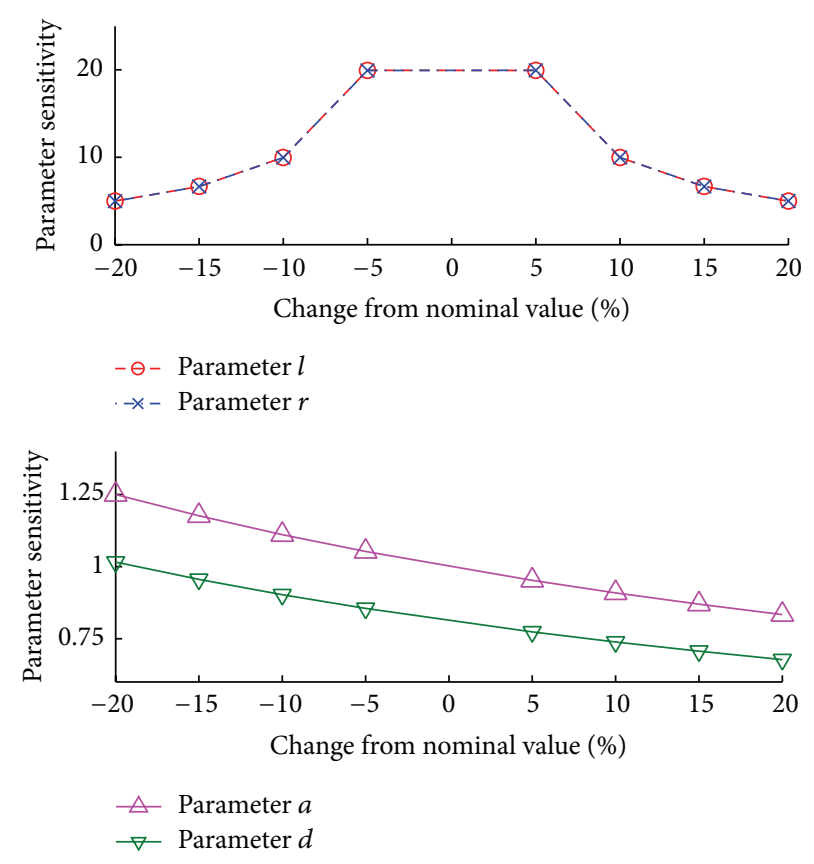

FIGURE 12: Sensitive of demagnetization speed point.

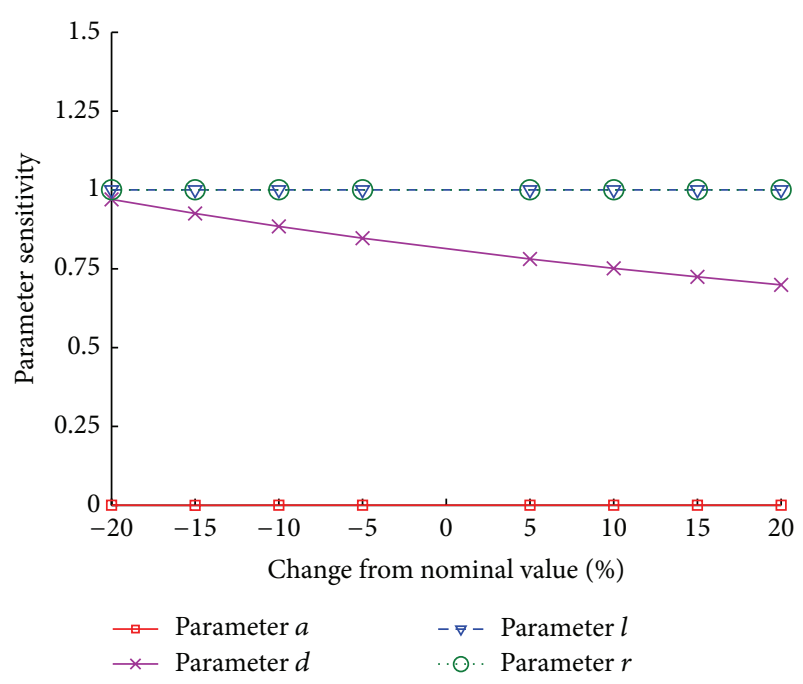

FIGURE 13: Sensitive of maximum braking torque.

based on the fundamental of the magnetic circle theory while introducing the concept of antimagnetic force. Furthermore, simulations on the braking torque characteristics were performed based on the developed ECB model, in which the influencing factors of torque generation properties were investigated by the variations of the parameters in ECB's structure design. By introducing the torque stability index, namely, the demagnetization speed point and the nominal maximum braking torque, the performance and influencing factors of ECB were studied, and the results are listed as follows.

(i) The stability of ECB's torque generation property could be enhanced by shifting the demagnetization 
TABle 3: Sensitivity of demagnetization speed point.

\begin{tabular}{lcccccccc}
\hline & $-20 \%$ & $-15 \%$ & $-10 \%$ & $-5 \%$ & $5 \%$ & $10 \%$ & $15 \%$ & $20 \%$ \\
\hline$a$ & 1.2500 & 1.1765 & 1.1111 & 1.0526 & 0.9524 & 0.9091 & 0.8696 & 0.8333 \\
$d$ & 1.0156 & 0.9559 & 0.9028 & 0.8553 & 0.7738 & 0.7386 & 0.7065 & 0.6771 \\
$l$ & 4.9874 & 6.6491 & 9.9724 & 19.9424 & 19.9376 & 9.9676 & 6.6442 & 4.9826 \\
$r$ & 4.9813 & 6.6431 & 9.9667 & 19.9368 & 19.9429 & 9.9727 & 6.6493 & 4.9875 \\
\hline
\end{tabular}

TABLE 4: Sensitivity of maximum braking torque.

\begin{tabular}{lcccccccc}
\hline & $-20 \%$ & $-15 \%$ & $-10 \%$ & $-5 \%$ & $5 \%$ & $10 \%$ & $15 \%$ & $20 \%$ \\
\hline$a$ & 1.0000 & 1.0000 & 1.0000 & 1.0000 & 1.0000 & 1.0000 & 1.0000 & 1.0000 \\
$d$ & $7.7 e-16$ & 0 & $1.5 e-15$ & $3.0 e-15$ & $3.0 e-15$ & $3.0 e-15$ & $1.0 e-15$ & $7.7 e-16$ \\
$l$ & 0.9701 & 0.9253 & 0.8844 & 0.8496 & 0.7808 & 0.7514 & 0.7242 & 0.6989 \\
$r$ & 1.0000 & 1.0000 & 1.0000 & 1.0000 & 1.0000 & 1.0000 & 1.0000 & 1.0000 \\
\hline
\end{tabular}

speed point, and the ECB's demagnetization speed point could be shifted by optimizing the internal parameters when design.

(ii) The most remarkable influencing factor on the shifting the demagnetization speed point of ECB was the thickness of the air-gap, followed by the radius of pole shoe's cross section area, the distance from the pole shoe center to the rotation center, and thickness of the rotator disc.

(iii) The nominal maximum braking torque of an ECB also could be optimized by changing the internal parameters. The most influencing factor on the nominal maximum braking torque was the radius of pole shoe's cross section area and the distance from the pole shoe center to the rotation center, followed by the thickness of the air-gap and thickness of the rotator disc.

\section{Conflict of Interests}

The authors declare no conflict of interests.

\section{Acknowledgments}

The authors gratefully acknowledge the Hubei Key Laboratory of Advanced Technology for Automotive Components, Hubei Collaborative Innovation Centre for Automotive Components Technology, and the "Plan-Dawn (CHEN GUANG JI HUA)” Funding for Youth Scientific and Technical Researchers by Wuhan Government (2013072304010830). In addition, the authors acknowledge the members from energy-plus innovation group for eco-vehicle technology of WUT.

\section{References}

[1] W. R. Smythe, "On eddy currents in a rotating disk," Transactions of the American Institute of Electrical Engineers, vol. 61, no. 9, pp. 681-684, 1942.

[2] J. G. Oetzel, "Eddy current retarder," SAE Technical Paper 540236, SAE, 1954.
[3] J. H. Wouterse, "Critical torque and speed of eddy current brake with widely separated soft iron poles," IEE Proceedings B: Electric Power Applications, vol. 138, no. 4, pp. 153-158, 1991.

[4] E. Simeu and D. Georges, "Modeling and control of an eddy current brake," Control Engineering Practice, vol. 4, no. 1, pp. 1926, 1996.

[5] K. Lee and K. Park, "Optimal robust control of a contactless brake system using an eddy current," Mechatronics, vol. 9, no. 6, pp. 615-631, 1999.

[6] D.-S. Li, W.-L. Yin, and K. Zhang, "Influence factor of braking torque in electromagnetic liquid-cooled retarder," Journal of Beijing University of Technology, vol. 6, pp. 831-836, 2014.

[7] R. He, C.-Y. Liu, and F.-Y. Yi, “Computation method for thermal field of eddy current retarder in automobile," Journal of Jiangsu University, vol. 26, no. 2, pp. 117-120, 2005.

[8] C. Y. Liu, K. J. Jiang, and Y. Zhang, "Design and use of an eddy current retarder in an automobile," International Journal of Automotive Technology, vol. 12, no. 4, pp. 611-616, 2011.

[9] L. Barnes, J. Hardin, and C. A. Gross, "An eddy current braking system," in Proceedings of the 25th Southeastern Symposium on System Theory (SSST '93), pp. 58-62, 1993.

[10] K. V. Tatis, A. G. Kladas, and J. A. Tegopoulos, "Geometry optimization of solid rotor eddy current brake by using sensitivity analysis and 3D finite elements," Journal of Materials Processing Technology, vol. 161, no. 1-2, pp. 363-367, 2005.

[11] R. K. Srivastava and S. Kumar, "An alternative approach for calculation of braking force of an eddy-current brake," IEEE Transactions on Magnetics, vol. 45, no. 1, pp. 150-154, 2009.

[12] J.-Y. Choi, H.-J. Shin, Y.-S. Park, and S.-M. Jang, "Torque analysis of axial flux PM type eddy current brake based on analytical field computations," in Proceedings of the International Conference on Electrical Machines and Systems (ICEMS '11), August 2011.

[13] R. He, X. Liu, and C. Liu, "Brake performance analysis of ABS for eddy current and electrohydraulic hybrid brake system," Mathematical Problems in Engineering, vol. 2013, Article ID 979384, 11 pages, 2013.

[14] O. Bottauscio, M. Chiampi, and A. Manzin, "Modeling analysis of the electromagnetic braking action on rotating solid cylinders," Applied Mathematical Modelling, vol. 32, no. 1, pp. 12-27, 2008. 
[15] A. Younis, K. Karakoc, Z. Dong, E. Park, and A. Suleman, "Application of SEUMRE global optimization algorithm in automotive magnetorheological brake design," Structural and Multidisciplinary Optimization, vol. 44, no. 6, pp. 761-772, 2011.

[16] H. Zhang, X. Guo, L. Xu et al., "Parameters analysis of hydraulicelectrical energy regenerative absorber on suspension performance," Advances in Mechanical Engineering, vol. 2014, Article ID 836502, 12 pages, 2014. 


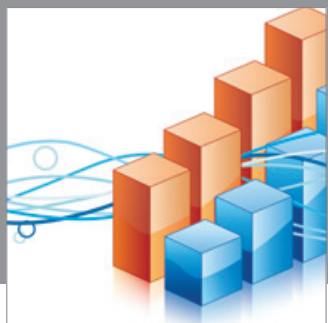

Advances in

Operations Research

mansans

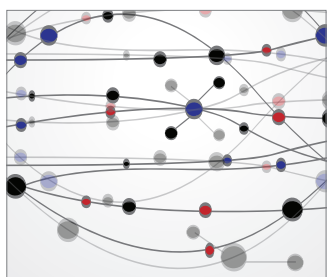

The Scientific World Journal
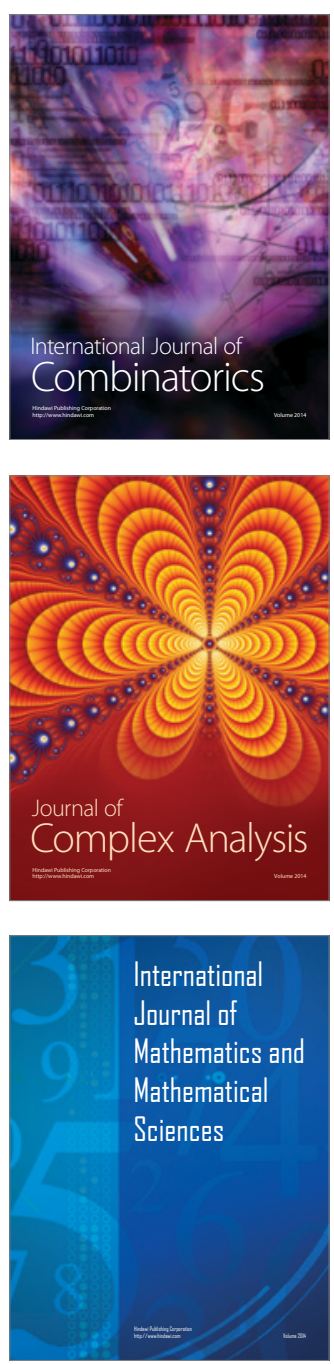
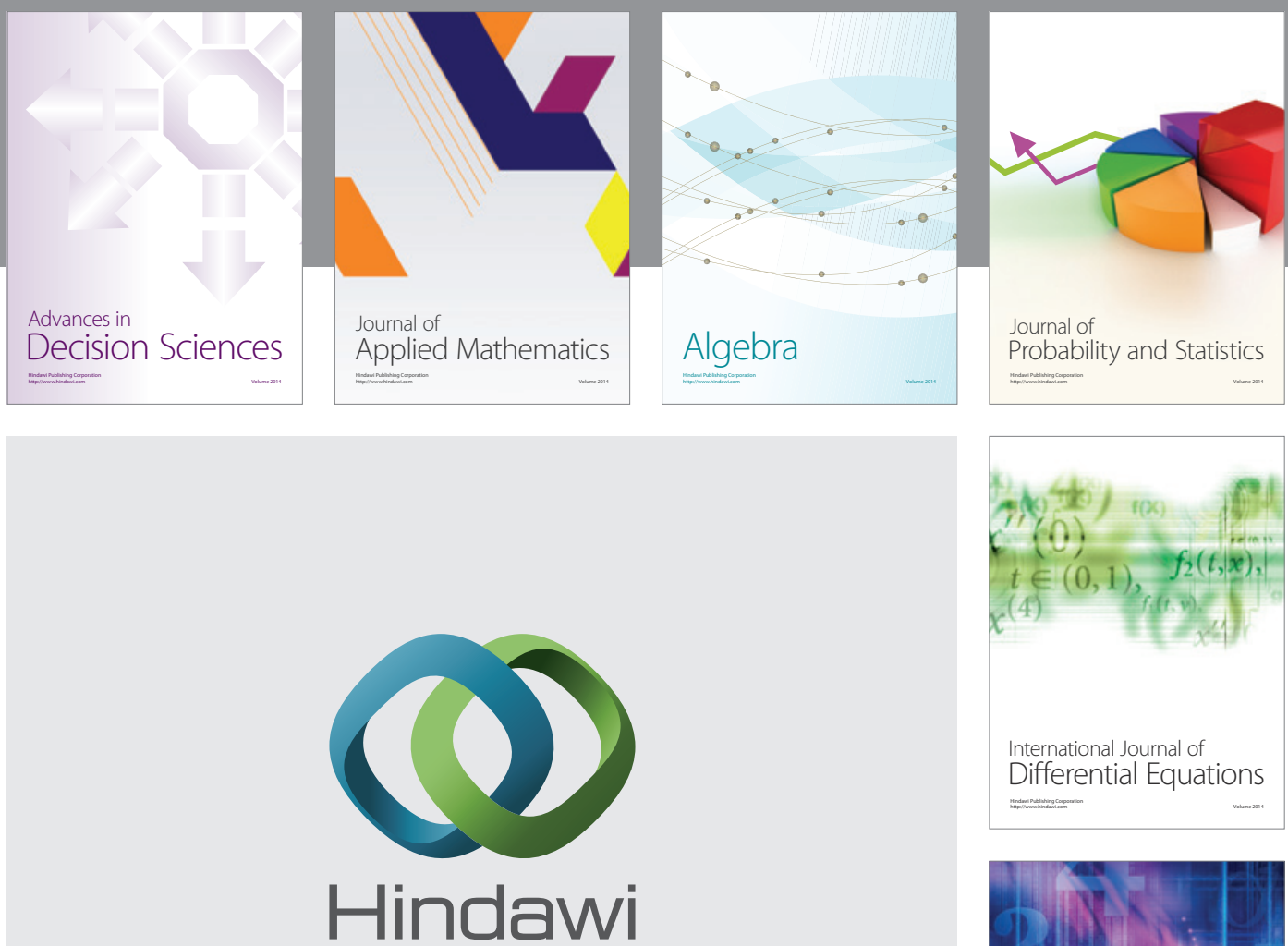

Submit your manuscripts at http://www.hindawi.com
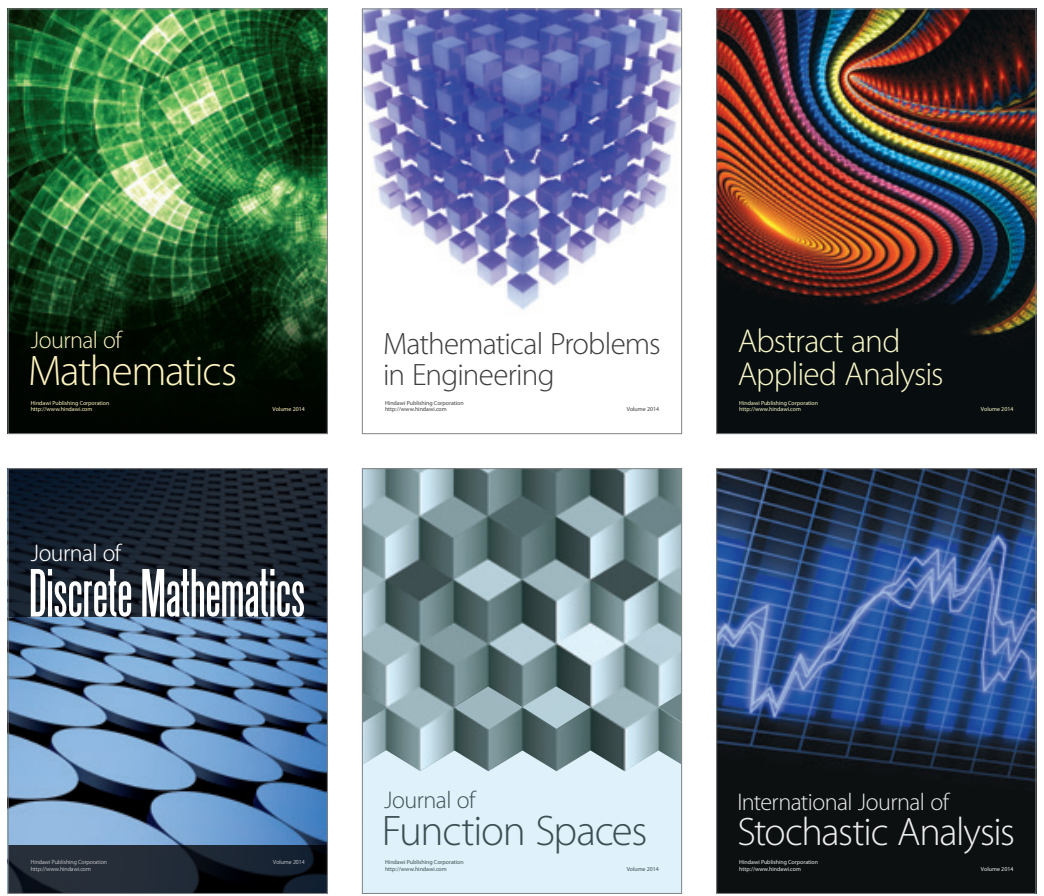

Journal of

Function Spaces

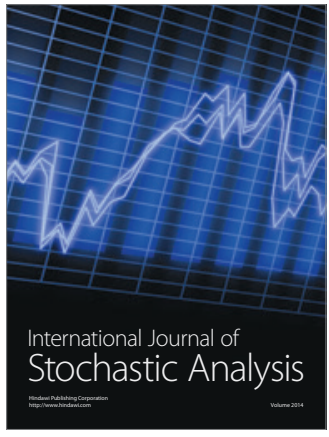

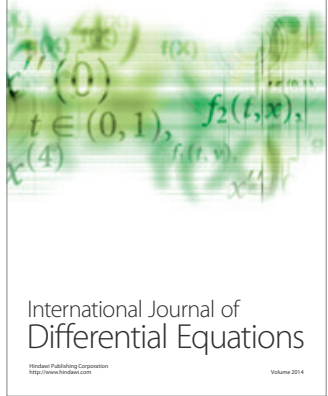
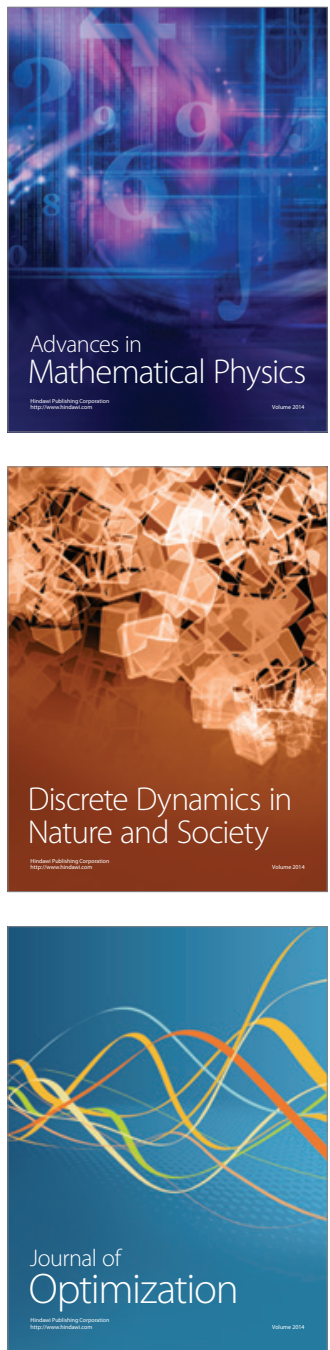